\title{
A Transformation of Carbonates into Sulfones at Benzylic Position via Palladium-Catalyzed Benzylic Substitution
}

Ryoichi Kuwano*, Yutaka Kondo, and Tsuyoshi Shirahama

Department of Chemistry, Graduate School of Sciences, Kyushu University, 6-10-1 Hakozaki, Higashi-ku, Fukuoka 812-8581, Japan

\section{Supporting Information}

General and Materials. NMR spectra were measured with Bruker AVANCE 400 (9.4 T magnet) spectrometer. IR spectra were measured with Nicolet Magna 560. Elemental analyses were performed by Service Centre of Elementary Analysis of Organic Compounds in Kyushu University. Flash column chromatographies and medium-pressure liquid chromatographies (MPLC) were performed with silica gel 60 (230-400 mesh, Merck) and with C. I. G. pre-packed column CPS-223L-1 (Kusano, Tokyo, Japan), respectively.

All reactions were conducted under nitrogen atomosphere. DMSO was distilled from calcium hydride under nitorogen. All benzylic carbonates were prepared from esterification of the corresponding alcohols with methyl chloroformate in the presence of $\mathrm{Et}_{3} \mathrm{~N}$, and were purified with flash column chromatography. $\quad\left[\mathrm{Pd}\left(\eta^{3}-\mathrm{C}_{3} \mathrm{H}_{5}\right) \mathrm{Cl}\right]_{2}$ was prepared according to literature, ${ }^{1}$ but was commercially available. DPEphos was purchased from STREM. Sodium sulfinates 1a-1d were purchased and were used without further purification.

General Procedure of Sulfonylation of Benzylic Carbonates with Sodium Arenesulfinates (Table 2). Under nitrogen atmosphere, benzylic methyl carbonate 1 (1.0 mmol) was added to a solution of $\left[\mathrm{Pd}\left(\eta^{3}-\mathrm{C}_{3} \mathrm{H}_{5}\right) \mathrm{Cl}\right]_{2}(1.8 \mathrm{mg}, 5.0 \mu \mathrm{mol})$ and DPEphos $(5.8 \mathrm{mg}, 11 \mu \mathrm{mol})$ in DMSO $(1.0 \mathrm{ml})$. After $5 \mathrm{~min}$, sodium sulfinate $2(1.1 \mathrm{mmol})$ was added to the solution under nitrogen stream. The mixture was stirred at $80{ }^{\circ} \mathrm{C}$ until 1 was completely consumed (monitored by GC). After $\mathrm{H}_{2} \mathrm{O}$ was added, the mixture was extracted several times with hexane or EtOAc. The combined organic layer was washed with brine, was dried with $\mathrm{Na}_{2} \mathrm{SO}_{4}$, and was evaporated under reduced pressure. The residue was purified by a flash column chromatography (EtOAc/hexane) or by MPLC (EtOAc/hexane) after passing through a short column on silica gel (EtOAc/hexane $=1 / 3-3 / 1)$ to give the desired product.

Benzyl phenyl sulfone ${ }^{2}$ (3a) (Scheme 1). The general procedure was followed with benzyl methyl carbonate (1a) $(167 \mathrm{mg}, 1.00 \mathrm{mmol})$ and sodium benzenesulfinate (2a) (181 $\mathrm{mg}, 1.10 \mathrm{mmol})$. The crude product was purified by a flash column chromatography $($ EtOAc/hexane $=1 / 1)$ to give 3a (221 mg, 95\%) as colorless solid: ${ }^{1} \mathrm{H}$ NMR (400 MHz, $\left.\mathrm{CDCl}_{3}, \mathrm{TMS}\right) \delta 4.31(\mathrm{~s}, 2 \mathrm{H}), 7.08(\mathrm{~d}, J=7.2 \mathrm{~Hz}$, 2H), $7.26(\mathrm{t}, J=7.3 \mathrm{~Hz}, 2 \mathrm{H}), 7.29-7.34(\mathrm{~m}, 1 \mathrm{H}), 7.45(\mathrm{t}, J=7.8 \mathrm{~Hz}, 2 \mathrm{H}), 7.57-7.66(\mathrm{~m}, 3 \mathrm{H}) ;{ }^{13} \mathrm{C}\left\{{ }^{1} \mathrm{H}\right\}$ NMR $\left(100 \mathrm{MHz} \mathrm{CDCl}_{3}\right) \delta 62.9,128.1,128.56,128.63,128.7,128.9,130.8,133.7,137.9$.

2-Methylbenzyl phenyl sulfone ${ }^{3}$ (3b) (Table 1, entry 1). The general procedure was followed with methyl 2-methylbenzyl carbonate (1b) $(179 \mathrm{mg}, 1.00 \mathrm{mmol})$ and $\mathbf{2 a}(181 \mathrm{mg}, 1.10 \mathrm{mmol})$. The crude product was purified by a flash column chromatography (EtOAc/hexane $=1 / 1)$ to give $\mathbf{3 b}(232$ $\mathrm{mg}, 95 \%$ ) as colorless solid: ${ }^{1} \mathrm{H} \mathrm{NMR}\left(400 \mathrm{MHz}, \mathrm{CDCl}_{3}, \mathrm{TMS}\right) \delta 2.11(\mathrm{~s}, 3 \mathrm{H}), 4.38(\mathrm{~s}, 2 \mathrm{H}), 7.02(\mathrm{~d}, J$ $=7.8 \mathrm{~Hz}, 1 \mathrm{H}), 7.07-7.13(\mathrm{~m}, 2 \mathrm{H}), 7.22(\mathrm{t}, J=7.5 \mathrm{~Hz}, 1 \mathrm{H}), 7.46(\mathrm{t}, J=7.8 \mathrm{~Hz}, 2 \mathrm{H}), 7.59-7.67(\mathrm{~m}, 3 \mathrm{H})$; ${ }^{13} \mathrm{C}\left\{{ }^{1} \mathrm{H}\right\} \mathrm{NMR}\left(100 \mathrm{MHz}, \mathrm{CDCl}_{3}\right) \delta 19.3,60.1,126.0,126.5,128.7,128.9,129.0,130.6,131.9,133.7$, 138.3, 138.4.

Phenyl 2,4,6-trimethylbenzyl sulfone (3c) (Table 1 entry 2). The general procedure was followed with methyl 2-methylbenzyl carbonate (1c) $(209 \mathrm{mg}, 1.00 \mathrm{mmol})$ and $\mathbf{2 a}(181 \mathrm{mg}, 1.10$ $\mathrm{mmol})$. The crude product was purified by a flash column chromatography $(\mathrm{EtOAc} / \mathrm{hexane}=1 / 1)$ to 
give 3c (244 mg, 89\%) as colorless solid: mp 130.5-131.5 ${ }^{\circ} \mathrm{C} ;{ }^{1} \mathrm{H}$ NMR (400 $\left.\mathrm{MHz}, \mathrm{CDCl}_{3}, \mathrm{TMS}\right) \delta$ $2.16(\mathrm{~s}, 6 \mathrm{H}), 2.26(\mathrm{~s}, 3 \mathrm{H}), 4.47(\mathrm{~s}, 2 \mathrm{H}), 6.84(\mathrm{~s}, 2 \mathrm{H}), 7.50(\mathrm{t}, J=7.8 \mathrm{~Hz}, 2 \mathrm{H}), 7.64(\mathrm{t}, J=7.5 \mathrm{~Hz}, 1 \mathrm{H})$, $7.77(\mathrm{~d}, J=7.5 \mathrm{~Hz}, 2 \mathrm{H}) ;{ }^{13} \mathrm{C}\left\{{ }^{1} \mathrm{H}\right\}$ NMR $\left(100 \mathrm{MHz}, \mathrm{CDCl}_{3}\right) \delta 20.2,21.0,57.3,122.2,128.4,129.1$, 129.4, 133.7, 138.4, 139.0, 139.6; IR (thin film) 1318, $1145 \mathrm{~cm}^{-1}$; Anal. Calcd for $\mathrm{C}_{16} \mathrm{H}_{18} \mathrm{O}_{2} \mathrm{~S}$ : C, 70.04; H, 6.61. Found: C, 70.01; H, 6.59.

4-Methoxybenzyl phenyl sulfone ${ }^{4}$ (3d) (Table 1, entry 3). The general procedure was followed with 4-methoxybenzyl methyl carbonate (1d) $(196 \mathrm{mg}, 1.00 \mathrm{mmol})$ and $\mathbf{2 a}(181 \mathrm{mg}, 1.10 \mathrm{mmol})$. The crude product was purified by a flash column chromatography (EtOAc/hexane $=1 / 1)$ to give 3d (232 $\mathrm{mg}, 89 \%$ ) as colorless solid: ${ }^{1} \mathrm{H}$ NMR (400 MHz, $\left.\mathrm{CDCl}_{3}, \mathrm{TMS}\right) \delta 3.79$ (s, 3H), 4.25 (s, 2H), 6.78 (d, $J$ $=8.7 \mathrm{~Hz}, 2 \mathrm{H}), 6.99(\mathrm{~d}, J=8.7 \mathrm{~Hz}, 2 \mathrm{H}), 7.46(\mathrm{t}, J=7.8 \mathrm{~Hz}, 2 \mathrm{H}), 7.57-7.66(\mathrm{~m}, 3 \mathrm{H}) ;{ }^{13} \mathrm{C}\left\{{ }^{1} \mathrm{H}\right\} \mathrm{NMR}$ $\left(100 \mathrm{MHz}, \mathrm{CDCl}_{3}\right) \delta 55.3,62.2,114.0,120.0,128.6,128.9,132.0,133.6,138.0,160.0$.

4-Methoxybenzyl 4-methylphenyl sulfone ${ }^{5}$ (3e) (Table 1, entry 4). The general procedure was followed with 1d (197 mg, $1.00 \mathrm{mmol})$ and sodium $p$-toluenesulfinate $\mathbf{2 b}(196 \mathrm{mg}, 1.10 \mathrm{mmol})$. The crude product was purified by MPLC (EtOAc/hexane $=1 / 1)$ after passing through a short column on silica gel $($ EtOAc/hexane $=1 / 1)$ to give $3 \mathrm{e}(263 \mathrm{mg}, 95 \%)$ as colorless solid: ${ }^{1} \mathrm{H}$ NMR $(400 \mathrm{MHz}$, $\left.\mathrm{CDCl}_{3}, \mathrm{TMS}\right) \delta 2.42(\mathrm{~s}, 3 \mathrm{H}), 3.79(\mathrm{~s}, 3 \mathrm{H}), 4.23(\mathrm{~s}, 2 \mathrm{H}), 6.79(\mathrm{~d}, J=8.7 \mathrm{~Hz}, 2 \mathrm{H}), 7.00(\mathrm{~d}, J=8.7 \mathrm{~Hz}$, $2 \mathrm{H}), 7.24(\mathrm{~d}, J=8.2 \mathrm{~Hz}, 2 \mathrm{H}), 7.51(\mathrm{~d}, J=8.2 \mathrm{~Hz}, 2 \mathrm{H}) ;{ }^{13} \mathrm{C}\left\{{ }^{1} \mathrm{H}\right\} \mathrm{NMR}\left(100 \mathrm{MHz}, \mathrm{CDCl}_{3}\right) \delta 21.6,55.3$, $62.2,114.0,120.2,128.6,129.5,132.0,135.1,144.5,159.9$.

4-Chlorobenzyl phenyl sulfone ${ }^{6}$ (3f) (Table 1, entry 5). The general procedure was followed with 4-chlorobenzyl methyl carbonate (1e) $(200 \mathrm{mg}, 0.99 \mathrm{mmol})$ and 2a $(181 \mathrm{mg}, 1.10 \mathrm{mmol})$. The crude product was purified by MPLC $($ EtOAc/hexane $=1 / 1)$ after passing through a short column on silica gel $($ EtOAc/hexane $=1 / 1)$ to give $3 f(238 \mathrm{mg}, 90 \%)$ as colorless solid: ${ }^{1} \mathrm{H} \mathrm{NMR}(400 \mathrm{MHz}$, $\left.\mathrm{CDCl}_{3}, \mathrm{TMS}\right) \delta 4.27(\mathrm{~s}, 2 \mathrm{H}), 7.02(\mathrm{~d}, J=8.4 \mathrm{~Hz}, 2 \mathrm{H}), 7.24(\mathrm{~d}, J=8.4 \mathrm{~Hz}, 2 \mathrm{H}), 7.48(\mathrm{t}, J=7.8 \mathrm{~Hz}, 2 \mathrm{H})$, 7.60-7.67 (m, 3H); ${ }^{13} \mathrm{C}\left\{{ }^{1} \mathrm{H}\right\}$ NMR $\left(100 \mathrm{MHz}, \mathrm{CDCl}_{3}\right) \delta 62.1,126.6,128.6,128.8,129.0,132.0,133.9$, 135.1, 137.7 .

4-(Methoxycarbonyl)benzyl phenyl sulfone ${ }^{7}$ (3g) (Table 1, entry 6). The general procedure was followed with 4-(methoxycarbonyl)benzyl methyl carbonate (1e) (225 $\mathrm{mg}, 1.00 \mathrm{mmol}$ ) and 2a (182 mg, $1.11 \mathrm{mmol}$ ). The crude product was purified by a flash column chromatography $($ EtOAc/hexane $=1 / 1)$ to give $\mathbf{3 g}(267 \mathrm{mg}, 92 \%)$ as colorless solid: ${ }^{1} \mathrm{H} \mathrm{NMR}\left(400 \mathrm{MHz}, \mathrm{CDCl}_{3}, \mathrm{TMS}\right)$ $\delta 3.92(\mathrm{~s}, 3 \mathrm{H}), 4.36(\mathrm{~s}, 2 \mathrm{H}), 7.16(\mathrm{~d}, J=8.3 \mathrm{~Hz}, 2 \mathrm{H}), 7.46(\mathrm{t}, J=7.8 \mathrm{~Hz}, 2 \mathrm{H}), 7.59-7.65(\mathrm{~m}, 3 \mathrm{H}), 7.93$ $(\mathrm{d}, J=8.3 \mathrm{~Hz}, 2 \mathrm{H}) ;{ }^{13} \mathrm{C}\left\{{ }^{1} \mathrm{H}\right\}$ NMR $\left(100 \mathrm{MHz}, \mathrm{CDCl}_{3}\right) \delta 52.3,62.7,128.6,129.0,129.7,130.5,130.8$, 133.1, 133.9, 137.6, 166.5.

(1-Naphthyl)methyl phenyl sulfone ${ }^{8}$ (3h) (Table 1, entry 7). The general procedure was followed with methyl (1-naphthyl)methyl carbonate $(\mathbf{1 g})(215 \mathrm{mg}, 0.99 \mathrm{mmol})$ and $\mathbf{2 a}(181 \mathrm{mg}, 1.10$ mmol). The crude product was purified by MPLC (EtOAc/hexane $=1 / 1$ ) after passing through a short column on silica gel (EtOAc/hexane $=1 / 1)$ to give $\mathbf{3 h}(266 \mathrm{mg}, 95 \%)$ as colorless solid: ${ }^{1} \mathrm{H}$ NMR (400 $\left.\mathrm{MHz}_{\mathrm{CDCl}}, \mathrm{TMS}\right) \delta 4.82(\mathrm{~s}, 2 \mathrm{H}), 7.20(\mathrm{~d}, J=7.0 \mathrm{~Hz}, 1 \mathrm{H}), 7.34(\mathrm{t}, J=7.9 \mathrm{~Hz}, 3 \mathrm{H}), 7.37-7.46(\mathrm{~m}$, $2 \mathrm{H}), 7.51(\mathrm{t}, J=7.0 \mathrm{~Hz}, 1 \mathrm{H}), 7.59(\mathrm{~d}, J=7.4 \mathrm{~Hz}, 2 \mathrm{H}), 7.79-7.84(\mathrm{~m}, 3 \mathrm{H}) ;{ }^{13} \mathrm{C}\left\{{ }^{1} \mathrm{H}\right\} \mathrm{NMR}(100 \mathrm{MHz}$, $\left.\mathrm{CDCl}_{3}\right) \delta 59.8,123.5,124.4,125.0,125.9,126.6,128.6,128.8,129.7,130.5,132.0,133.6,133.7$, 137.9.

4-Chlorophenyl (1-naphthyl)methyl sulfone (3i) (Table 1, entry 8). The general procedure was followed with $\mathbf{1 g}(222 \mathrm{mg}, 1.03 \mathrm{mmol})$ and sodium $p$-chlorobenzenesulfinate (2c) (220 mg, 1.11 mmol). The crude product was purified by MPLC (EtOAc/hexane $=2 / 1$ ) after passing through a short column on silica gel (EtOAc/hexane $=2 / 1)$ to give 3i $(260 \mathrm{mg}, 80 \%)$ as colorless solid: $\mathrm{mp}$ 156.9-157.9 ${ }^{\circ} \mathrm{C} ;{ }^{1} \mathrm{H}$ NMR (400 MHz, $\left.\mathrm{CDCl}_{3}, \mathrm{TMS}\right) \delta 4.83(\mathrm{~s}, 2 \mathrm{H}), 7.21(\mathrm{~d}, J=7.0 \mathrm{~Hz}, 1 \mathrm{H}), 7.30(\mathrm{~d}, J$ $=8.6 \mathrm{~Hz}, 2 \mathrm{H}), 7.37(\mathrm{t}, J=7.7 \mathrm{~Hz}, 1 \mathrm{H}), 7.40-7.51(\mathrm{~m}, 4 \mathrm{H}), 7.79(\mathrm{~d}, J=8.3 \mathrm{~Hz}, 1 \mathrm{H}), 7.81-7.87(\mathrm{~m}$, $2 \mathrm{H}) ;{ }^{13} \mathrm{C}\left\{{ }^{1} \mathrm{H}\right\} \mathrm{NMR}\left(100 \mathrm{MHz}, \mathrm{CDCl}_{3}\right) \delta 59.9,123.4,125.0,126.0,126.7,128.7,129.1,129.9,130.2$, 
130.6, 131.9, 133.7, 136.3, 140.5; IR (thin film) $1151 \mathrm{~cm}^{-1}$; Anal. Calcd for $\mathrm{C}_{17} \mathrm{H}_{13} \mathrm{O}_{2} \mathrm{ClS}$ : C, 64.45; $\mathrm{H}$, 4.14. Found: C, 64.52; H, 4.15.

Methyl (1-naphthyl)methyl sulfone9 (3j) (Table 1, entry 9). The general procedure was followed with $1 \mathbf{g}(212 \mathrm{mg}, 0.98 \mathrm{mmol})$ and sodium methanesulfinate (2d) (118 $\mathrm{mg}, 1.16 \mathrm{mmol})$. The crude product was purified by MPLC (EtOAc/hexane $=1 / 1)$ after passing through a short column on silica gel $($ EtOAc/hexane $=1 / 1)$ to give $3 \mathbf{j}(216 \mathrm{mg},>99 \%)$ as colorless solid: ${ }^{1} \mathrm{H} \mathrm{NMR}(400 \mathrm{MHz}$, $\left.\mathrm{CDCl}_{3}, \mathrm{TMS}\right) \delta 2.76(\mathrm{~s}, 3 \mathrm{H}), 4.78(\mathrm{~s}, 2 \mathrm{H}), 7.49-7.65(\mathrm{~m}, 4 \mathrm{H}), 7.92(\mathrm{dd}, J=4.0,8.0 \mathrm{~Hz}, 2 \mathrm{H}), 8.11(\mathrm{~d}, J$ $=8.5 \mathrm{~Hz}, 1 \mathrm{H}) ;{ }^{13} \mathrm{C}\left\{{ }^{1} \mathrm{H}\right\} \mathrm{NMR}\left(100 \mathrm{MHz}, \mathrm{CDCl}_{3}\right) \delta 39.5,58.3,123.5,124.6,125.4,126.3,127.2,129.1$, $130.1,130.4,131.8,134.1$.

5-Methoxy-2-methylbenzyl phenyl sulfone ${ }^{10}(3 k)$ (Table 1, entry 10). The general procedure was followed with 5-methoxy-2-methylbenzyl methyl carbonate (1h) (212 mg, $1.01 \mathrm{mmol})$ and $\mathbf{2 a}$ (186 $\mathrm{mg}, 1.13 \mathrm{mmol}$ ) in the presence of $2 \mathrm{~mol} \%$ of DPEphos-palladium catalyst. The crude product was purified by MPLC (EtOAc/hexane $=1 / 1$ ) after passing through a short column on silica gel (EtOAc/hexane $=1 / 1)$ to give $3 \mathbf{k}(215 \mathrm{mg}, 77 \%)$ as pale yellow needles: ${ }^{1} \mathrm{H} \mathrm{NMR}\left(400 \mathrm{MHz}, \mathrm{CDCl}_{3}\right.$, TMS) $\delta 2.07(\mathrm{~s}, 3 \mathrm{H}), 3.77(\mathrm{~s}, 3 \mathrm{H}), 4.31(\mathrm{~s}, 2 \mathrm{H}), 6.62-6.67(\mathrm{~m}, 2 \mathrm{H}), 6.94(\mathrm{~d}, J=8.1 \mathrm{~Hz}, 1 \mathrm{H}), 7.46(\mathrm{t}, J$ $=7.8 \mathrm{~Hz}, 2 \mathrm{H}), 7.62(\mathrm{t}, J=7.5 \mathrm{~Hz}, 1 \mathrm{H}), 7.66(\mathrm{~d}, J=7.8 \mathrm{~Hz}, 2 \mathrm{H}) ;{ }^{13} \mathrm{C}\left\{{ }^{1} \mathrm{H}\right\} \mathrm{NMR}\left(100 \mathrm{MHz}, \mathrm{CDCl}_{3}\right) \delta$ 19.6, 55.1, 59.5, 111.4, 116.0, 118.5, 128.6, 128.9, 133.1, 133.6, 138.4, 139.8, 159.9 .

3-Amino-5-(tert-butyldiphenylsilyloxy)benzyl phenyl sulfone ${ }^{11}$ (3l) (Table 1, entry 11). The general procedure was followed with 3-amino-5-(tert-butyldiphenylsilyloxy)benzyl methyl carbonate (1i) $(81 \mathrm{mg}, 0.186 \mathrm{mmol})$ and $2 \mathbf{a}(36 \mathrm{mg}, 0.219 \mathrm{mmol})$ in the presence of $5 \mathrm{~mol} \%$ of DPEphos-palladium catalyst. The crude product was purified by a flash column chromatography $($ EtOAc/hexane $=1 / 1)$ to give $31(67 \mathrm{mg}, 72 \%)$ as pale yellow oil: ${ }^{1} \mathrm{H}$ NMR $\left(400 \mathrm{MHz}, \mathrm{CDCl}_{3}, \mathrm{TMS}\right) \delta$ $1.03(\mathrm{~s}, 9 \mathrm{H}), 3.42$ (br s, 2H), $4.04(\mathrm{~s}, 2 \mathrm{H}), 5.84-5.86(\mathrm{~m}, 1 \mathrm{H}), 5.96(\mathrm{t}, J=2.0 \mathrm{~Hz}, 1 \mathrm{H}), 6.02-6.04(\mathrm{~m}$, $1 \mathrm{H}), 7.34-7.66(\mathrm{~m}, 15 \mathrm{H}) ;{ }^{13} \mathrm{C}\left\{{ }^{1} \mathrm{H}\right\}$ NMR $\left(100 \mathrm{MHz}, \mathrm{CDCl}_{3}\right) \delta 19.4,26.5,62.8,106.8,110.6,112.7$, $127.7,128.6,128.7,129.8,129.9,132.8,133.4,135.4,137.9,147.4,156.4$.

\section{References}

1. Tatsuno, Y.; Yoshida, T.; Otsuka, S. Inorg. Synth. 1979, 19, 220.

2. $\quad$ Chen, Y.; Lam, Y.; Lai, Y.-H. Org. Lett. 2003, 5, 1067-1069.

3. Wildeman, J.; van Leusen, A. M. Synthesis 1979, 733-734.

4. $\quad$ Lai, S.; Lee, D. G. Tetrahedron 2002, 58, 9879-9887.

5. Yau, E. K.; Coward, J. K. J. Org. Chem. 1990, 55, 3147-3158.

6. Jarvis, B. B.; Saukaitis, J. C. J. Am. Chem. Soc. 1973, 95, 7708-7715.

7. DeGraw, J. I.; Christie, P. H.; Brown, E. G.; Kelly, L. F.; Kisliuk, R. L.; Gaumont, Y.; Sirotnak, F. M. J. Med. Chem. 1984, 27, 376-380.

8. Satoh, T.; Kondo, A.; Musashi, J. Tetrahedron 2004, 60, 5453-5460.

9. Arnold, B.; Donald, L.; Jurgens, A.; Pincock, J. A. Can. J. Chem. 1985, 63, 3140-3146.

10. Taber, D. F.; Jiang, Q.; Chen, B.; Zhang, W.; Campbell, C. L. J. Org. Chem. 2002, 67, 4821-4827.

11. Smith, A. B., III; Barbosa, J.; Wong, W.; Wood, J. L. J. Am. Chem. Soc. 1996, 118, 8316-8328. 